\title{
Comportamentos autolesivos na adolescência e disfunção familiar: relato de caso
}

Vânia M. Duarte,* Mário Machado Cruz,** Bertínia Oliveira***

\section{RESUMO}

Introdução: Os comportamentos autolesivos (CAL) são uma realidade prevalente na população adolescente. São sinal de adolescência patológica, existindo múltiplos fatores de risco para CAL, como a disfunção familiar e a doença mental parental. Com este caso pretende-se demonstrar como os cuidados de saúde primários (CSP) permitem uma orientação adequada das situações de CAL e de disfunção familiar, devido à sua acessibilidade e capacidade de articulação com outros recursos.

Descrição do caso: NV, sexo masculino, 12 anos, recorreu à consulta por episódio de CAL (cortes nos antebraços com uma tesoura), associado a irritabilidade, impulsividade e agressividade verbal, na sequência de comportamento agressivo do pai. Foi referenciado ao serviço de urgência de pedopsiquiatria e agendada nova consulta, assegurando o seguimento adequado em articulação com os cuidados de saúde secundários (CSS). Constatou-se a presença de disfunção familiar, com repercussões no desenvolvimento do adolescente. Procurou-se realizar intervenção nos vários membros da família. Houve adesão do NV às consultas de pedopsiquiatria e de psicologia, com melhoria dos sintomas descritos.

Comentário: O papel do MF é fundamental na deteção precoce dos CAL e na sua orientação. Neste caso foi possível intervir oportunisticamente, aquando do pedido de ajuda, o que demonstra a importância da acessibilidade nos CSP e da sua abordagem multissistémica e familiar.

Palavras-chave: Disfunção Familiar; Adolescência; Médico de Família; Comportamento Autolesivo.

\section{INTRODUÇÃO}

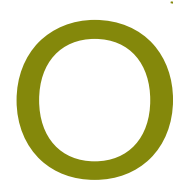

s comportamentos autolesivos (CAL) correspondem a um profundo mal-estar e nunca devem ser desvalorizados. São sempre sinal de adolescência patológica, na qual não estão a ser cumpridas as tarefas essenciais desta etapa do desenvolvimento. Os CAL correspondem, muitas vezes, à porção oculta do “icebergue", sendo um fator de risco para suicídio. A prevalência de CAL encontrada em Portugal foi de 15,6\% em 2010. ${ }^{1}$ Existem múltiplos fatores de risco para CAL, entre os quais se encontram a disfunção familiar e a doença mental parental. $^{2}$

O médico de família (MF) tem a oportunidade de exercer a sua profissão de uma forma única, ao poder trabalhar com o indivíduo em toda a sua circunstância.

*Médica Assistente de Medicina Geral e Familiar, UCSP Tapada das Mercês, ACES Sintra

**Médico Interno de Medicina Geral e Familiar. USF AlphaMouro, ACES Sintra

***Médica Assistente de Medicina Geral e Familiar, USF Albasaúde, ACES Sintra
Cabe ao MF estar atento aos sinais de alerta que façam suspeitar de disfunção familiar. Com este caso pretende-se demonstrar como os cuidados de saúde primários (CSP) permitem uma orientação adequada das situações de CAL e disfunção familiar, devido à sua acessibilidade e capacidade de articulação com outros recursos.

Este caso clínico mostra como a disfunção familiar é vivenciada por um adolescente, comprometendo o seu desenvolvimento e bem-estar, contribuindo para a prática de CAL. Pretendemos também salientar a importância do MF na gestão dos problemas no seio familiar e o seu papel no auxílio do reequilíbrio do sistema família.

\section{DESCRIÇÃO DO CASO}

NV, adolescente do sexo masculino de 12 anos de idade, caucasiano, natural da Amadora, residente na Rinchoa, a repetir o sexto ano de escolaridade. Pertence a uma família nuclear na fase $\mathrm{V}$ do ciclo de vida de Duvall e na Classe III da escala de Graffar, 
sendo o terceiro filho de uma fratria de três irmãos. Sociável, gostando da escola que frequenta e com boa relação com colegas e professores. Nega bullying. Sem história de consumos de substâncias. Nos tempos livres frequenta os escuteiros e gosta de jogar computador.

Tem história de episódios de convulsões febris, com seguimento em consulta de neurologia pediátrica até aos sete anos de idade, por história de epilepsia paterna. Apresentou um seguimento irregular nas consultas de vigilância de saúde infantil, tendo frequentado apenas as consul-

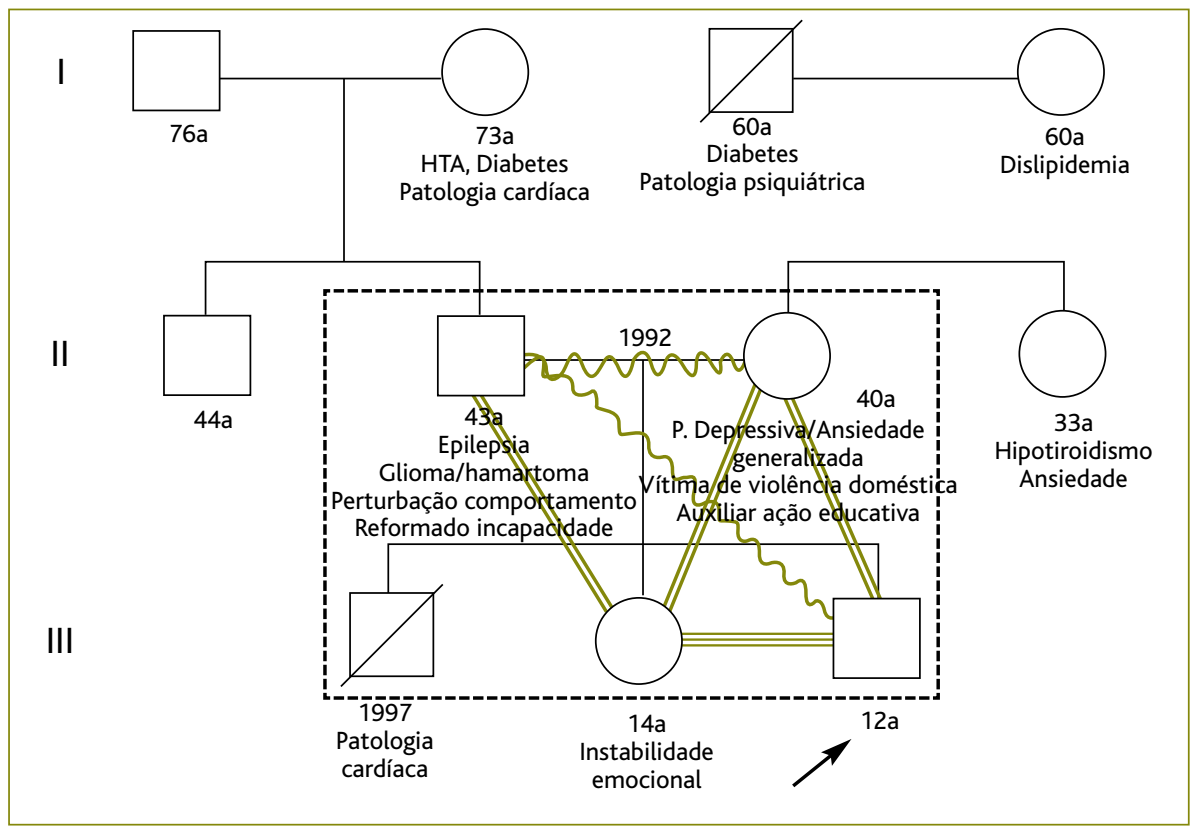

Figura 1. Genograma familiar com psicofigura de Mitchell (realizado a 12 de outubro de 2012). tas: um mês, dois meses, quatro meses, dois anos e cinco anos. Salienta-se ainda a mudança de MF e de unidade de saúde nessa altura. Tem excesso de peso (índice de massa corporal no percentil 85).

A mãe apresenta patologia depressiva e ansiedade generalizada desde há 13 anos, altura em que foi feito o diagnóstico de epilepsia ao marido. Recorre a múltiplas consultas por sintomas inespecíficos com difícil adesão às terapêuticas propostas. O pai é seguido em consulta de neurologia por epilepsia (de difícil controlo) e tumor cerebral em estudo. O casal teve um primeiro filho, que faleceu aos três meses de idade, há 16 anos, por patologia cardíaca. A tia materna sofre de "ansiedade" (sic) e o avô materno tinha patologia do foro psiquiátrico não especificada (ver figura 1).

\section{Julho de 2012}

Recorreu à nossa consulta, trazido pela mãe, por episódio de CAL (cortes nos antebraços com uma tesoura). A mãe refere, no último ano, grande irritabilidade, impulsividade e agressividade verbal por parte do filho. Quando questionado sobre os motivos desse comportamento, o adolescente revelou uma grande infelicidade e revolta em relação ao comportamento paterno. O pai profere frases como "vais ser maricas",

"és gordo" (sic). A mãe do adolescente refere ainda que o marido "sempre implicou" (sic) com o filho mas que, no último ano, houve um agravamento, tornando-se mais agressivo verbalmente. Para além da violência psicológica praticada no seio familiar, adotou comportamentos de agressividade física para com vizinhos e pessoas estranhas, dos quais resultaram duas queixas na polícia. Na sequência desta consulta, foi feita referenciação ao serviço de urgência de pedopsiquiatria e agendada nova consulta para a semana seguinte, para fazer o ponto da situação.

\section{Agosto de 2012}

A mãe compareceu sozinha à consulta de NV, referindo que este já havia sido avaliado em pedopsiquiatria, com plano terapêutico e de seguimento estruturado. Sem novos CAL e com melhoria das queixas de irritabilidade e impulsividade.

\section{Outubro de 2012}

Na sequência da consulta de exame global de saúde foi averiguada a adesão às consultas nos cuidados de saúde secundários. Nesta família impunha-se a realização de um estudo de avaliação familiar pelos seguintes motivos: doença crónica paterna, problemas emo- 


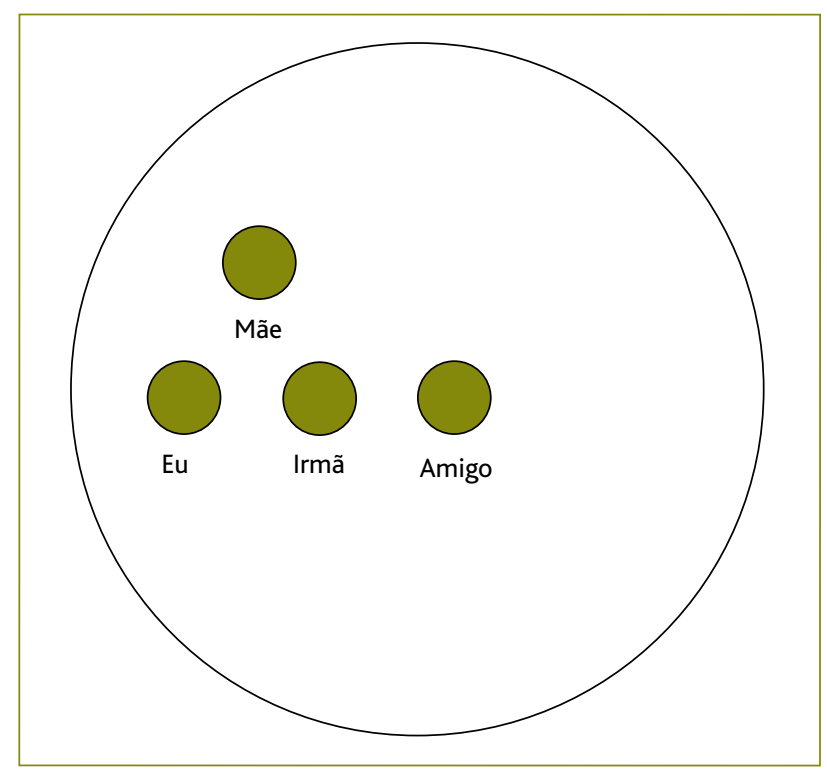

Figura 2. Círculo de Thrower do NV (realizado a 12 de outubro de 2012).

cionais e comportamentais graves no seio familiar, doenças na fase de transição do ciclo de vida, elevado consumo de consultas por sintomas inespecíficos, com fraca adesão à terapêutica por parte da mãe e morte na família. ${ }^{4}$

Foram, assim, realizadas duas consultas separadas: uma ao NV e outra à mãe e foram aplicados o genograma, com psicofigura de Mitchell, o círculo de Thrower e o APGAR Familiar de Smilkstein. Durante a construção do genograma ficámos a saber que os pais do NV tinham tido um primeiro filho que morreu aos três meses de idade e o adolescente revela que o pai

\section{QUADRO I. Lista de problemas}

\section{Lista de Problemas}

Perturbação do comportamento - comportamento autolesivo

Vítima de abuso psicológico

Doença e comportamento do pai

Patologia depressiva e ansiedade da mãe

Relação conflituosa entre pai e filho

Violência doméstica lhe diz "quem devia estar cá era o outro filho e não ele" (sic). Este sofrimento psicológico acompanhou o NV durante vários anos, levando-o a sentir-se culpado pela situação e a castigar-se a ele próprio, automutilando-se. Da análise da psicofigura de Mitchell (figura 1) destaca-se o conflito conjugal e o conflito existente entre pai e filho. Quando questionada a mãe sobre a sua relação conjugal, esta refere comportamentos de desconfiança e ciúme por parte do marido, na sequência do agravamento da sua doença neurológica. O APGAR Familiar de Smilkstein aplicado ao NV apresenta uma pontuação de 2, compatível com uma família com disfunção acentuada. ${ }^{4}$

Nas consultas subsequentes tentou-se conhecer melhor os seus hábitos de vida e gostos pessoais. Revelou-se um adolescente bastante reservado, respondendo a perguntas abertas com um simples "não sei” (sic) ou limitando-se, muitas vezes, a monossílabos. Desabafou com a irmã sobre a sua relação com o pai e com uma amiga que conheceu na rede social facebook, com a qual se identifica por também ter CAL.

Face ao descrito, torna-se pertinente elaborar uma lista de problemas (quadro I) para execução do plano subsequente.

\section{PLANO E EVOLUÇÃO \\ Pai}

Convidou-se o pai do adolescente a comparecer à consulta, com o intuito de averiguar a sua relação com os outros membros da família, a sua situação clínica, adesão à terapêutica e articulação com os CSS. O pai não compareceu.

\section{Adolescente}

Uma vez que o adolescente foi visto na urgência de pedopsiquiatria e encaminhado para a consulta da mesma especialidade, procurou-se mostrar disponibilidade para receber o NV sempre que necessitasse e confirmar a adesão às consultas. Tentou-se articulação com a pedopsiquiatra e com a psicóloga, as quais não responderam ao documento redigido pelo MF.

\section{Mãe}

Identificando a mãe como a gestora dos problemas neste seio familiar, mostrou-se abertura para a receber, ajudando-a e capacitando-a para esta tarefa. Por ini- 
ciativa própria, a mãe ativou a Comissão de Proteção de Crianças e Jovens em Risco e iniciou o processo de divórcio.

\section{Irmã}

Convidou-se a irmã a vir à nossa consulta, da qual se apurou instabilidade emocional e dificuldades no sucesso escolar. Passou a ser seguida em consulta do adolescente (realizada pela interna do seu MF) e em consulta de psicologia.

\section{COMENTÁRIO}

Nas crianças e adolescentes saudáveis, por vezes é difícil o diagnóstico precoce de situações de sofrimento psicológico. Por um lado e por iniciativa própria, os adolescentes procuram pouco os cuidados de saúde; por outro, os pais tendem a marcar menos consultas à medida que a criança vai crescendo. ${ }^{2}$ Para além disso, por vezes também é difícil manter para os pais o seguimento da criança em dois locais diferentes, CSP e CSS. Neste caso destacam-se a mudança de MF, de unidade de saúde e ainda a não comparência às consultas pelo NV. Tal pode explicar a dificuldade na deteção mais precoce da perturbação do comportamento do adolescente e de disfunção familiar.

Esta família era prioritária para a realização de um estudo de avaliação familiar. Foram aplicados os seguintes métodos: genograma com psicofigura de Mitchell, APGAR Familiar de Smilkstein e círculo de Thrower. O genograma revelou-se útil na documentação dos antecedentes familiares de doença do foro mental, mas principalmente na aquisição da informação da morte do primeiro filho do casal, desconhecida até à data pelo MF. A psicofigura de Mitchell permitiu identificar a perceção do adolescente acerca das relações entre os vários elementos familiares, evidenciando a conflitualidade no seio familiar. Optou-se por aplicar o círculo de Thrower ao NV devido à sua dificuldade em falar das relações familiares. No entanto, este não revelou informação adicional para a elaboração do plano, o que levanta a questão da utilidade da sua aplicação neste caso.

A autoagressão é uma realidade com uma prevalência assinalável na população adolescente. ${ }^{5} \mathrm{O}$ MF deve estar sensibilizado para os sinais e sintomas de alarme de CAL e a sua orientação e articulação precoce. Nesta família salientam-se, como fatores de risco, violência doméstica psicológica, doença mental parental, discussões maritais frequentes, dificuldades no relacionamento entre os membros da família e impulsividade do adolescente.

O papel do MF é fundamental na deteção precoce destes comportamentos e na orientação de situações passíveis de correção que afetem negativamente quer o adolescente quer a família. Neste caso foi possível intervir oportunisticamente aquando do pedido de ajuda na sequência do CAL, em consulta de doença aguda, o que demonstra a importância da acessibilidade nos CSP. A partir deste momento foi estabelecida uma relação médico-doente, até à altura não existente, com um aumento de confiança, empatia e o estabelecimento de um plano terapêutico.

\section{AGRADECIMENTOS}

À Dra . Susana Medeiros, pela sua disponibilidade e inspiração.

\section{REFERÊNCIAS BIBLIOGRÁFICAS}

1. Matos MG. Health Behaviour in School-age Children (HBSC), dados nacionais 2010-Abril 2011: problemas emergentes e contextos sociais. Lisboa: Aventura Social; 2011.

2. Monteiro P. Psicologia e psiquiatria da infância e adolescência. Lisboa: LIDEL; 2014. ISBN 9789897520372

3. Caeiro RT. Registos clínicos em medicina familiar. Lisboa: Instituto de Clínica Geral da Zona Sul; 1991.

4. Rebelo L. A família em medicina geral e familiar: conceitos e práticas. Lisboa: Verlag Dashöfer; 2011. ISBN 9789896421939

5. Evans $P$, editor. A definição europeia de medicina geral e familiar (clínica geral/medicina familiar) [Internet]. Barcelona:WONCA Europa; 2002. Available from: http://www.woncaeurope.org/sites/default/files/documents/European\%20Definition\%20in\%20Portuguese.pdf

6. Sampaio D, Resina T. Família: saúde e doença. Lisboa: Instituto de Clínica Geral da Zona Sul; 1994.

\section{CONFLITO DE INTERESSES}

Os autores declaram não ter conflitos de interesses.

\section{ENDEREÇO PARA CORRESPONDÊNCIA}

Vânia M. Duarte

E-mail: vaniamduarte21@gmail.com

Recebido em 01-11-2014

Aceite para publicação em 19-11-2015 


\section{ABSTRACT}

\section{DELIBERATE SELF-HARM IN ADOLESCENCE AND FAMILY DYSFUNCTION: CASE REPORT}

Introduction: Deliberate self-harm (DSH) has a significant prevalence among young people. It is a sign of disturbed adolescence. There are multiple risk factors, including family dysfunction and parental mental illness. This case report shows how the family doctor, with good accessibility and communication with other health resources, can guide the patient and the family in situations of DSH and family dysfunction.

Case description: NV, a 12 year-old male, was brought to our clinic after an episode of self-harm (cutting his forearms with scissors), irritability, impulsivity, and verbal aggression, following aggressive behavior by his father. The family doctor referred the boy to a child psychiatry emergency service. A second appointment was scheduled to ensure adequate follow-up together with secondary care. Family dysfunction was diagnosed with repercussions for adolescent development. Several family members received treatment. The adolescent agreed to frequent child psychiatry and psychology consultations, with improvement in symptoms.

Commentary: The family doctor plays an essential role in the early detection and referral of cases of DSH. In this case, opportunistic intervention, when the adolescent first sought help, shows the importance of accessibility and the systemic family approach.

Keywords: Family Dysfunction; Adolescence; Family Doctor; Deliberate Self-harm. 